\title{
THE SCHMIDT TELESCOPE AT CALAR ALTO OBSERVATORY
}

\author{
K. BIRKLE, H. BELLEMAN and H. ELSÄSSER \\ Max-Planck Institut für Astronomie \\ D-69117 Heidelberg \\ Koenigstuhl 17 \\ Germany
}

The Schmidt telescope at Calar Alto Observatory, Spain, was installed there in 1980 by the MaxPlanck-Institut für Astronomie, Heidelberg, and has been operated since then by this institute; originally it had been operational at the Hamburg Observatory since 1955 . The corrector plate and mirror have $80 \mathrm{~cm}$ and $120 \mathrm{~cm}$ diameter respectively; focal length is $240 \mathrm{~cm}$. A field 5.5 deg by $5.5 \mathrm{deg}$ is photographed on $24 \mathrm{~cm}$ by $24 \mathrm{~cm}$ curved plates. Two objective prisms of 1.7 deg and $4.0 \mathrm{deg}$ refractive angle are available (Heckmann 1955; Birkle 1984). Thousands of direct and spectral plates have been taken, mainly for the Hamburg Quasar Survey (Engels et al. 1988). Other observing programs referred e.g. to astrometry of comets, giving support to ESA's Giotto space encounters with comets Halley and Grigg-Skjellerup (Roeser et al. 1986; Morley 1992). Gradually, however, the number of plates increased showing image quality which did not correspond to the excellent performance of the telescope optics. Several technical improvements have therefore been installed to remove some obvious mechanical instability.

For diagnostic purposes, a video camera centred in front of the corrector plate was used to measure position variations of the mirror, plate holder, and corrector plate relative to each other due to bending effects of the tube structure when the telescope was inclined to any position. Down to zenith distances of $60 \mathrm{deg}$, only small bending up to a few millimeters was observed, not exceeding the corresponding optical tolerances. Moreover, bending showed good symmetry relative to the zenith and relative to the 3 invar rods by which the distance between mirror and plate holder is kept constant independently of temperature changes. Thus, up to now, the tube structure has maintained its original quality.

Because of being mechanically worn out after many years of use, a complete new fixing device for the plate holder and glass filter in the tube had to be constructed along with a new focusing mechanism and new plate holders. Loading, locking and unlocking the plate holder and filter inside the tube are now controlled and carried out semi-automatically. Locking the plate holder is always done by the same motor-driven force, avoiding personal handling for sake of precision. The lids of the new plate holders have a central locking mechanism, by which the lid with its spherical inner surface is locked at all 4 sides of the plate holder simultaneously, thus pressing the photographic plate uniformly into its curved position. This results in a better adaption of the curved plate to an ideal spherical surface, i.e. more uniform focusing over the whole plate. Using a 3-D-coordinate measuring machine (accuracy 2 micron), the rms deviation of the curved plate surface from a sphere was found to be $< \pm 15$ micron, i.e. much less than the focus tolerance of \pm 35 micron for 1 arcsec seeing.

\section{6}

H. T. MacGillivray et al. (eds.), Astronomy from Wide-Field Imaging, 46-48. (C) 1994 IAU. Printed in the Netherlands. 
As the Kodak price for a standard sized 8 by 10 inch plate is much lower than for a $24 \mathrm{~cm}$ by $24 \mathrm{~cm}$ plate, we also built 8 by 10 inch plate holders. A rectangular plate spherically bent will show more deviations from a sphere than a square plate. Yet, with the central locking mechanism, the 3-D-measurements showed for the central part of about $12 \mathrm{~cm}$ diameter of a rectangular plate the same small rms deviation as for a square plate; towards the plate edges the deviations still remained within the above focus tolerance.

Both guiding telescopes $(\mathrm{f} / 15, \mathrm{f}=300 \mathrm{~cm})$ have been equipped with $\mathrm{CCD}$ autoguiders commercially available as SBIG ST4. The guide star to be used can first be selected and positioned through the eyepiece, then it will be focused on the CCD chip of the ST4. The limiting magnitude is about 11th mag, sufficient for a field $1 \mathrm{deg}$ by $1 \mathrm{deg}$, accessible with the movable eyepiece-CCD carriage at each guiding telescope. Experience with these autoguiders is very satisfactory: easy handling and sharper images.

With the new plate holder system and the autoguiders the number of plates of high image quality increased considerably. E.g. in samples of 285 and 146 spectral plates taken for the Hamburg Quasar Survey before and after these improvements, respectively, the percentage of plates of the best quality class, defined essentially by the FWHM of the spectra perpendicular to the dispersion, has increased by a factor of more than 4 (D. Groote, 1992, private communication).

As examples of the attainable astrometric accuracy, 1) the rms value of 0.56 arcsec for both the R.A. and Decl. residuals of comet Halley's 99 position measurements on Calar Alto Schmidt plates is mentioned, taken over a period of 12 months before the Giotto encounter (Bastian et al. 1986; Morley 1986). This compares with twice this rms for the total of measurements selected from all IHW observations. 2) Comet Grigg-Skjellerup, the second target of the Giotto space mission, was more difficult to observe owing to its unfavourable visibility conditions from Calar Alto and its relative faintness. The comet was observed with CCD cameras at the other Calar Alto telescopes. For astrometry, Schmidt plates were taken using PPM and Carlsberg stars as primary standards, the CCD observations were linked to this system. The 16 Grigg-Skjellerup positions obtained at Calar Alto between 9th September 1991 and 24th June 1992 have rms residuals of 0.56 arcsec in R.A. and 0.48 arcsec in Dec. as compared to the corresponding values 1,14 and 0.90 arcsec, respectively, for the total of 104 positions observed in the 10 months before the Giotto encounter (Morley 1992). Although observing conditions had been more difficult for this comet, due to the technical improvements realised after the Halley observations and due to the more precise PPM standard stars, the results were at least of the same accuracy as those of the Halley campaign.

\section{Acknowledgements}

Our special thanks go to U. Groezinger, MPIA Heidelberg, G. Lingenfelder and W. Mueller, Calar Alto. We also thank our collaborators of the precision mechanics department of the MPIA. 


\section{References}

Bastian, U., Birkle, K., Klare, G. and Roeser, S., 1986. Stern und Weltraum, 25, 638.

Birkle, K., 1984. 'Astronomy with Schmidt type Telescopes' Proc. 78th IAU Colloq., ed. M. Capaccioli, Reidel, Dordrecht, p. 203.

Engels, D., Groote, D., Hagen, H.J. and Reimers, D., 1988. Astron. Soc. Pac. Conf., 2, 143.

Heckmann, O., 1955. Mitt. Astron. Ges., 1954, 57.

Morley, T.A., 1986. Giotto Flight Dynamics Report, ESOC Darmstadt.

Morley, T., 1992. Proc. 43rd Congr. of the IAF, Washington.

Roeser, S., Bastian, U., Birkle, K. and Klare, G., 1986. Proc. 20th ESLAB Symp. on the Exploration of Halley's Comet, Heidelberg, ESA SP-250, p. 283. 